\title{
A hydrologic post-processor for ensemble streamflow predictions
}

\author{
L. Zhao ${ }^{1}$, Q. Duan ${ }^{2}$, J. Schaake ${ }^{3, *}$, A. $\mathbf{Y e}^{2}$, and J. Xia ${ }^{1}$ \\ ${ }^{1}$ Institute of Geographic Sciences and Natural Resources Research, Chinese Academy of Sciences, Beijing, China \\ ${ }^{2}$ College of Global Change and Earth System Science, Beijing Normal University, Beijing, China \\ ${ }^{3}$ NOAA/National Weather Service, Silver Spring, MD, USA \\ * retired
}

Received: 15 July 2010 - Revised: 3 October 2010 - Accepted: 3 October 2010 - Published: 28 February 2011

\begin{abstract}
This paper evaluates the performance of a statistical post-processor for imperfect hydrologic model forecasts. Assuming that the meteorological forecasts are wellcalibrated, we employ a "General Linear Model (GLM)" to post-process simulations produced by a hydrologic model. For a particular forecast date, the observations and simulations from an "analysis window" and hydrologic model forecasts for a "forecast window", the GLM Post-Processor (GLMPP) is used to produce an ensemble of predictions of the streamflow observations that will occur during the "forecast window". The objectives of the GLMPP are to: (1) preserve any skill in the original hydrologic ensemble forecast; (2) correct systematic model biases; (3) retain the equallikelihood assumption for the ensemble; (4) preserve temporal scale dependency relationships in streamflow hydrographs and the uncertainty in the predictions; and, (5) produce reliable ensemble predictions.

Observed and simulated daily streamflow data from the Second Workshop on Model Parameter Estimation Experiment (MOPEX) are used to test how well these objectives are met when the GLMPP is applied to ensemble hydrologic forecasts driven by well calibrated meteorological forecasts. A 39-year hydrologic dataset from the French Broad basin is split into calibration and verification periods. The results show that the GLMPP built using data from the calibration period removes the mean bias when applied to hydrologic model simulations from both the calibration and verification periods. Probability distributions of the post-processed model simulations are shown to be closer to the climatological probability distributions of observed streamflow than the distributions of the unadjusted simulated flows. A number of
\end{abstract}

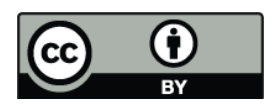

Correspondence to: Q. Duan

(qyduan@bnu.edu.cn) experiments with different GLMPP configurations were also conducted to examine the effects of different configurations for forecast and analysis window lengths on the robustness of the results.

\section{Introduction}

In an ensemble streamflow prediction (ESP) system, hydrologic model forecasts driven by forecasts of likely future meteorological events are commonly used to represent the likely future hydrologic events. The reliability and accuracy of hydrologic predictions are negatively affected by a number of sources, including unreliable and inaccurate meteorological forcing, initial basin state conditions, model errors (i.e., incorrect model structure and parameter specification), and errors in streamflow observations (McEnery et al., 2005; Schaake et al., 2006). In the ESP system like the US National Weather Service River Forecast System (Seo et al., 2006; DeMargne et al., 2009), these uncertainties are handled by different statistical processors (Fig. 1). For example, meteorological forcing uncertainty is handled by the ESP pre-processor (EPP). The uncertainty related to initial and boundary conditions is commonly addressed by land data assimilators. Uncertainty in the hydrologic simulations (i.e., the raw ensemble) is expected to be handled by an ESP postprocessor (EPostP).

A lot of recent effort has been directed at the development of different statistical processors. Krzysztofowicz and Sigrest (1999) developed methods to calibrate probabilistic quantitative precipitation forecast (PQPF) so PQPF is more consistent with observations. Seo et al. (2000) employed a quasi-analytical downscaling procedure to produce short-term probabilistic river stage forecasting using PQPF

Published by Copernicus Publications on behalf of the European Geosciences Union. 


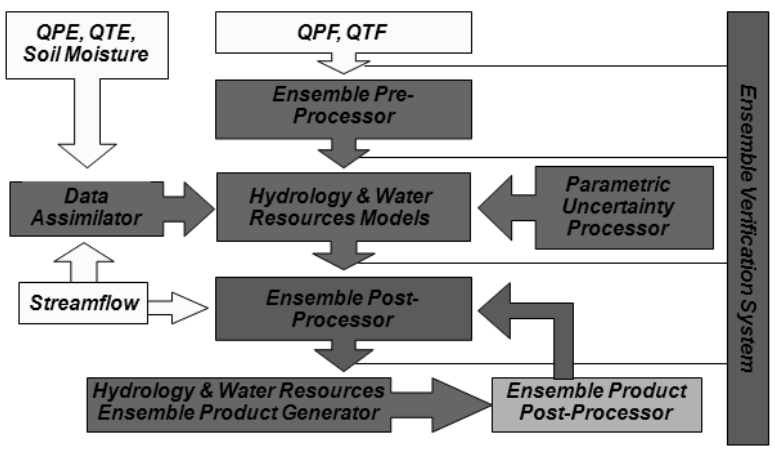

Fig. 1. A schematic of the ensemble streamflow prediction (ESP) system. QPE and QTE stand for quantitative precipitation estimation and quantitative temperature estimation, respectively, while QPF and QTF stand for quantitative precipitation forecast and quantitative temperature forecast.

from raw quantitative precipitation forecasts (QPF). Schaake et al. (2007) developed a methodology to map singlevalued QPF and QTF (i.e., quantitative temperature forecast) into calibrated ensemble forecasts. Schaake's methodology works by computing the marginal distribution of observation conditioned on a given forecast with a meta-Gaussian model and then using a procedure known as "Schaake Shuffle" to create space-time consistent meteorological ensembles (Clark et al., 2004).

Forecast updating through data assimilation (these can be experience-driven manual updating procedures or automated mathematical algorithms) serve to reduce model biases by adjusting model state variables and enhance forecast performance. With advances in observational technology, many researchers have been using sophisticated data assimilation techniques to obtain more realistic land state variables by assimilating observed data such as soil moisture, snow cover, or river discharge data. Seo et al. (2003) used the variational assimilation technique to assimilate observed precipitation and streamflow data to improve operational streamflow forecasts. Slater and Clark (2006) and Andreatis and Lettenmaier (2006) used Ensemble Kalman Filter (EnsKF) to assimilate observed snow data to improve the estimates of snow accumulation and melting. There exist a plethora of methodologies to deal with uncertainty related to model parameters (Duan et al., 1992, 2003, 2006). The most widely used approach to reduce parameter uncertainty is to calibrate model parameters to match hydrologic simulations with observations (Duan et al., 1992). Recent development in parameter estimation is to treat model parameters as probabilistic values to account for uncertainties in forcing and streamflow observations (Beven and Binley, 1992; Kuczera and Parent, 1998; Vrugt et al., 2003).
The traditional ESP generates an ensemble of streamflow forecasts conditioned on an ensemble of precipitation and temperature forecasts. The uncertainty in the initial conditions is typically ignored in the hydrologic model. This usually results in biased raw hydrologic simulations and the spread of the raw hydrologic ensemble tends to be underestimated, especially in the early forecast time steps where initial conditions and effects of hydrologic model uncertainty are most important. Post-processing is a way to remove biases in ensemble means and spread of ensemble streamflow predictions. Krzysztofowicz and Maranzano (2004) proposed a Bayesian based methodology to generate probabilistic river stage forecasting as a way to remove model biases. Based on this methodology, Seo et al. (2006) presented a hydrologic post-processor for the National Weather Service River Forecast System. Regianni et al. (2009) applied the same methodology to generate ensemble streamflow predictions for operational River Rhine forecasting system. Theoretically speaking, the Bayesian framework can explicitly integrate uncertainties from all sources (Krzysztofowicz, 2001; Seo et al., 2006). To do that, however, requires knowing the underlying multivariate probability distributions of these uncertainties, including their covariance structure. In reality, it is very difficult to know what forms those probability distributions take and what the covariance structure is.

Wood and Schaake (2008) correlated seasonal flow forecast ensemble means with observations to generate a conditional forecast mean and spread that lie between the climatological mean and spread (when the forecast has no skill) and the raw forecast mean with zero spread (when the forecast is perfect). A promising approach to post-processing hydrologic model hydrograph simulations using a transformation derived with simulated and observed flow hydrographs was proposed by Bogner and Kalas (2008). They used a combination of state-space models and wavelet transformations in order to update errors between the simulated (forecasted) and the observed discharge.

The work of Bogner and Kalas (2008) together with an understanding of the first and second moment properties of any multivariate distribution that was presented by Valencia and Schaake (1973) motivated the work presented here. In this work, a the normal quantile transform (NQT) using climatological distributions of observed and simulated streamflow variables is used to transform these variables to a space where all variables are assumed to be distributed according to a Bivariate Normal distribution that is completely characterized by its covariance matrix. Recent past streamflow observations and corresponding model simulations together with traces of future model streamflow values are used to predict an ensemble of future streamflow observations that are an estimate of the multi-scale predictive uncertainty of the future Streamflow. We will show that this procedure, called a General Linear Model Post-Processor (GLMPP) may be a useful tool for post-processing ensemble streamflow predictions. 


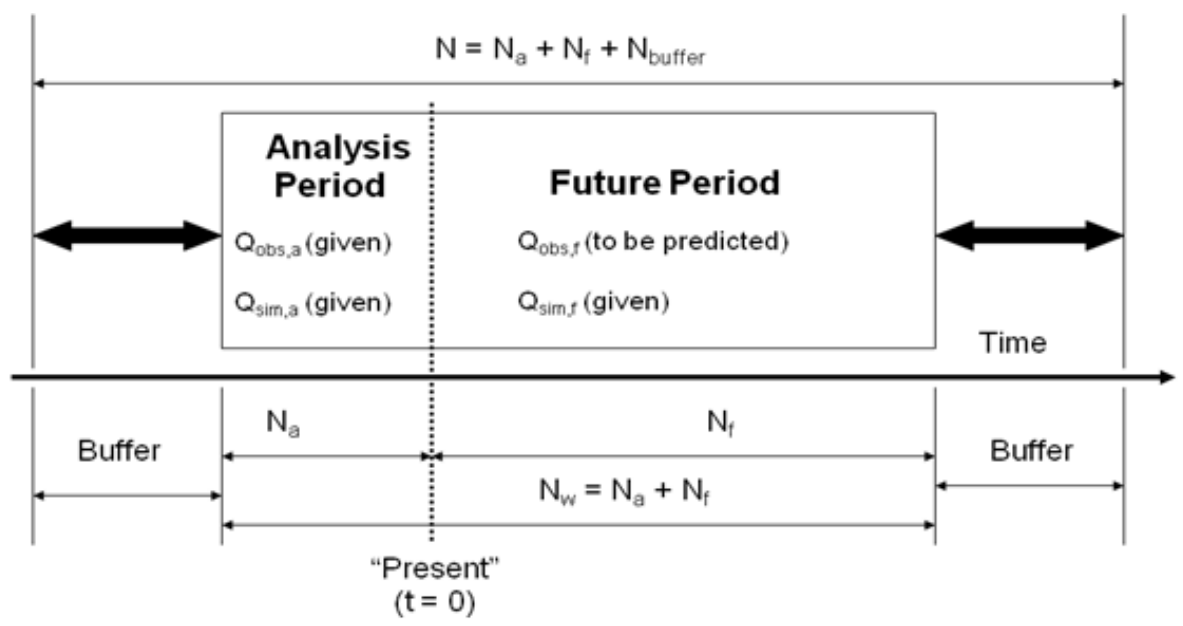

Fig. 2. The data window for the GLMPP.

The organization of the paper is as follows. The GLMPP methodology is presented in Sect. 2. The data and experimental configurations are described in Sect. 3. Section 4 discusses the experimental results. Section 5 provides conclusions.

\section{The general linear model methodology}

In this section we present a hydrologic post-processor with the following properties: (1) it preserves the "skill" of the raw ensemble forecast; (2) it removes mean bias; (3) it produces an ensemble of members that represent in an "equallylikely" sense the observed hydrograph that is being predicted; (4) it preserves temporal scale dependency relationships in streamflow hydrographs and the uncertainty in the predictions; and, (5) it produces ensemble predictions of future streamflow events that have very nearly the same climatology as the corresponding streamflow observations.

In probability terms, the function of a hydrologic postprocessor is to obtain the conditional probability density function (PDF) of observations, $y_{\mathrm{obs}}$, given the ensemble of meteorological forecasts, $y_{\mathrm{fcst}}, f\left(y_{\mathrm{obs}} \mid y_{\mathrm{fcst}}\right)$. If we neglect the uncertainty in the relationship between observations $\left(y_{\text {obs }}\right)$ and simulations $\left(y_{\text {sim }}\right)$ that is caused by the analysis error (i.e., the error in computing areal values from station values) in the estimated forcing used to generate $y_{\text {sim }}$ during the analysis period, $f\left(y_{\text {obs }} \mid y_{\text {fcst }}\right)$ can be estimated by the relationship:

$f\left(y_{\mathrm{obs}} \mid y_{\mathrm{fcst}}\right)=\int_{0}^{+\infty} f\left(y_{\mathrm{obs}} \mid y_{\mathrm{sim}}\right) f\left(y_{\mathrm{sim}} \mid y_{\mathrm{fcst}}\right) d y_{\mathrm{sim}}$

If random variables $y_{\mathrm{obs}}, y_{\mathrm{sim}}$ and $y_{\mathrm{fcst}}$ are Gaussian, Eq. (1) can then be solved using what we refer to as a General Linear Model Post-Processor (GLMPP). Before presenting the GLMPP, we first define some terms. Figure 2 is a schematic of the data window which specifies the day of forecast, the analysis period and the forecast period. $N_{a}$ and $N_{f}$ are the lengths of the analysis and forecast periods. Differences between observed and simulated flows for the analysis period preceding the forecast are used to compensate for unwanted effects of imperfect estimates of initial conditions on forecasts. Because there are typically only a few historical years of data available to calibrate the GLMPP it is essential to use observed and simulated flow data for forecast dates for some number of days before and after the target forecast date. Therefore a "buffer" period with the half length of $N_{\text {buffer }}$ is introduced to include data for a total of $N_{\text {buffer }}$ days prior to the analysis period and after the forecast period for each historical year to calibrate GLMPP. Let $Z_{1}$ be the observations for the forecast period, $Z_{2}$ the predictor vector, which contains simulated and observed streamflow for the analysis period, and simulated streamflow for the forecast period, i.e.,

$$
Z_{1}=\left[q_{\mathrm{obs}, n_{f}}\right]=\left[\begin{array}{l}
q_{\mathrm{obs}, 1} \\
\vdots \\
q_{\mathrm{obs}, N_{f}}
\end{array}\right], \quad Z_{2}=\left[\begin{array}{l}
q_{\mathrm{sim}, n_{f}} \\
q_{\mathrm{obs}, n_{a}} \\
q_{\mathrm{sim}, n_{a}}
\end{array}\right]=\left[\begin{array}{l}
q_{\mathrm{sim}, 1} \\
\vdots \\
q_{\mathrm{sim}, N_{f}} \\
q_{\mathrm{obs},-N_{a}} \\
\vdots \\
q_{\mathrm{obs},-1} \\
q_{\mathrm{sim},-N_{a}} \\
\vdots \\
q_{\mathrm{sim},-1}
\end{array}\right]
$$

Further, let $Z_{1,2}$ be the predictand given the predictor vector, i.e., $Z_{1,2}=Z_{1} \mid Z_{2}$. The GLMPP model can be expressed as:

$Z_{1.2}=A \cdot Z_{2}+B \cdot E$

where $E=N(0,1)$ is a normally distributed random variable with a mean of zero and a standard deviation of 1 . Denote 
$Z=\left[\begin{array}{l}Z_{1} \\ Z_{2}\end{array}\right]$. The covariance matrix for $Z$ is:

$\Sigma=\left[\begin{array}{ll}\Sigma_{11} & \Sigma_{12} \\ \Sigma_{21} & \Sigma_{22}\end{array}\right]$

Eq. (2) can be solved analytically (Valencia and Schaake, 1973) with $A=\Sigma_{12} \cdot \Sigma_{22}^{-1}$ and $B B^{T}=\Sigma_{11}-\Sigma_{12} \cdot \Sigma_{22}^{-1} \cdot \Sigma_{21}$.

Although these equations for $A$ and $B B^{T}$ apply to any multivariate distribution the GLMPP makes the additional assumption that the random variables $Z_{1}$ and $Z_{2}$ are normally distributed. Clearly, observed and simulated streamflow variables do not meet this requirement so we use the normal quantile transformation (NQT) proposed by Krzysztofowicz (1997) to transform elements of the streamflow data $Q_{\text {sim }}$ and $Q_{\mathrm{obs}}$ in original space to $q_{\mathrm{sim}}$ and $q_{\mathrm{obs}}$ in the transformed space where the marginal distributions of the transformed variables have a standard normal distribution. We then approximate the joint distribution of the normally distributed transformed variables with the Multivariate Normal distribution. To use estimates of $q_{\text {obs }}$ made in the transformed space, an inverse NQT procedure must be performed to transform $q_{\text {obs }}$ back into the original space.

\section{Data and experimental design}

The data used to test GLMPP are the simulated and observed daily streamflow discharge data obtained from Second Workshop on Model Parameter Estimation Experiment (MOPEX) database (Duan et al., 2006). There are 12 basins in the MOPEX database, but we selected only one of the MOPEX basins, the French Broad River basin at Asheville, North Carolina (USGS Basin ID 03451500) as an example to illustrate the proposed GLMPP approach. The drainage area is $2448 \mathrm{~km}^{2}$. Seventy-three percent of the drainage area is covered by forests. Annual precipitation is about $1676 \mathrm{~mm}$. There are a total of 39 years of streamflow data covering the period from 1960 to 1998 . The streamflow data period is further divided into calibration and verification periods, with the odd-year data used for calibrating the parameters of GLMPP, and the even-year data for verifying them. The reason for using odd and even years to represent calibration and verification periods is to ensure that the statistical properties of both periods are similar.

Based on former study, simulations from three of the hydrologic models that participated in MOPEX are chosen for this study: the Sacramento Soil Moisture Accounting (SACSMA) model (a detailed description of the SAC-SMA structure and parameters can be found in Burnash et al., 1973); the Simple Water Balance (SWB; Schaake et al., 1996) and the NOAH model (Chen and Dudhia, 2001). The SAC model with 16 adjustable parameters is the most widely used operational hydrologic forecast model in the National Weather Service. The SWB model, while also an operational model, has only 5 adjustable parameters. The NOAH model is a land surface parameterization scheme for the operational numerical weather prediction (NWP) model in NWS. These three models represent different levels of hydrologic forecast skills. Since our objective is to test if GLMPP applied to each model can meet the requirements for a hydrologic post-processor specified at the beginning of the Sect. 2, we designed a number of numerical experiments to perform the following studies: (1) we calibrated GLMPP to the streamflow data from all three models using data for a "calibration period" and then checked to see if the results from calibration period are still valid in and independent "verification period"; (2) we experimented with different configurations for the data window lengths to examine the robustness of the results; and (3) we tested different forecast dates to see if GLMPP performance is seasonally dependent.

\section{Results and discussions}

\subsection{Testing GLMPP on the SAC, SWB and NOAH streamflow forecasts}

In the first experiment, we separately calibrated GLMPP for the SAC, SWB and NOAH models using MOPEX streamflow forecast and observation data. The configuration of the data window is defined as: $N_{a}=15$ days, $N_{f}=30$ days and $N_{\text {buffer }}=60$ days. The forecast date is set to 15 February. Figure 3 shows how the means of the raw unadjusted ensemble and GLMPP adjusted ensemble compare with the observed means. Figure 3 clearly shows that the adjusted ensemble means obtained using GLMPP agree with the observations much better than those of the raw ensemble means in both the calibration and verification periods. Table 1 summarizes the root-mean-square (RMS) errors of the raw and GLMPP adjusted ensemble means. Figure 4 displays the mean standard deviations of observations, the raw ensemble, GLMPP adjusted ensemble for the three hydrologic models. It is obvious again that GLMPP adjusted ensemble shows much better agreement with the observations in both the calibration and verification periods. Figure 5 exhibits the cumulative distribution function (CDF) curves of the raw, GLMPP adjusted and observed ensembles for three hydrological models. This figure indicates that CDF curves based on the GLMPP adjusted ensemble are closer to the observed CDF than the raw ensemble for both calibration and verification periods. The results presented above suggest that GLMPP works well for all three models regardless of the original forecast skill levels. In other words, the poor forecast made a hydrologic model can be reasonably corrected by a well designed hydrologic post-processor. 
Table 1. RMS errors of raw and GLMPP adjusted ensemble means.

\begin{tabular}{lllllll}
\hline & SAC_raw & SAC_adj & NOAH_raw & NOAH_adj & SWB_raw & SWB_adj \\
\hline Calibration & 0.7488 & 0.0519 & 1.4285 & 0.0048 & 7.0576 & 0.0301 \\
Verification & 0.9084 & 0.0472 & 1.6567 & 0.0325 & 0.5644 & 0.0179 \\
\hline
\end{tabular}

(a)

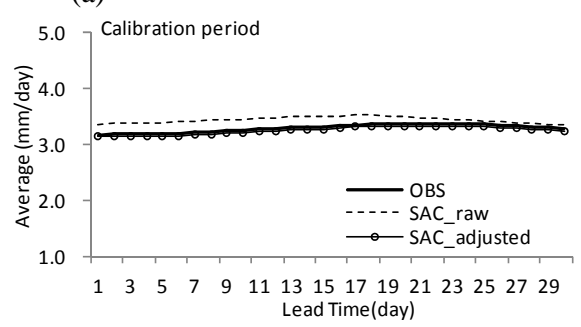

(d)

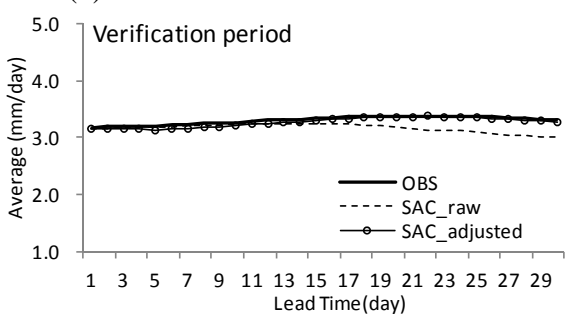

(b)

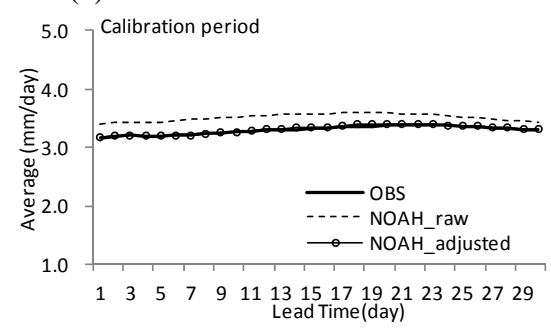

(e)

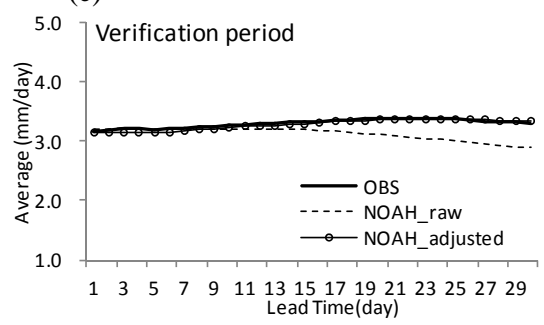

(c)

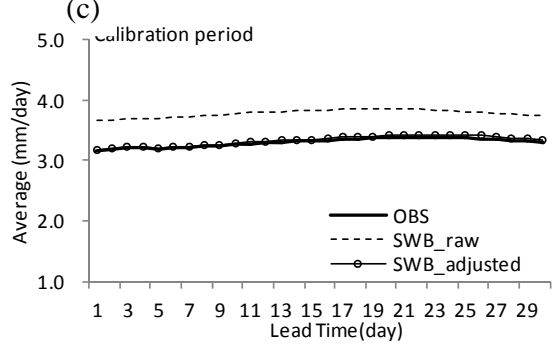

(f)

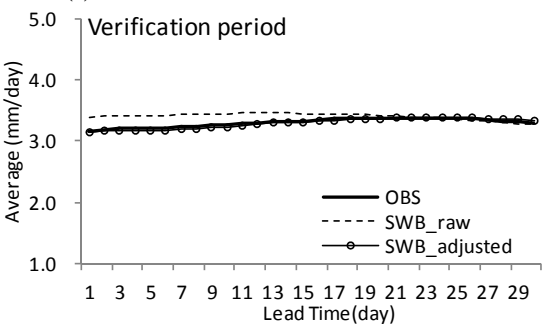

Fig. 3. The means of observed ensemble, raw ensemble, and GLMPP adjusted ensemble for the SAC, SWB and NOAH models.

\subsection{Testing GLMPP with different settings for the data window}

It is apparent that the results in Sect. 4.1 indicate that GLMPP does possess some of the desired properties for a hydrologic post-processor specified in Sect. 2. In this section, we conduct an experiment to examine the effects of different settings for the data window. The data for the SAC model is used for this experiment. The forecast date is set to February $15 . N_{a}$ takes on the value of 10,15 , or 20 days; $N_{f} 15$, 30 or 45 days; and $N_{\text {buffer }} 10,30$ or 60 days. We vary one parameter at a time, while the other parameters are kept at their default values. The default settings for these parameters are: $N_{a}=15$ days, $N_{f}=30$ days, and $N_{\text {buffer }}=60$ days. Figure 6 shows the impact of different settings on the agreement between the means from GLMPP adjusted ensembles and observed means for both calibration and verification periods. Figure $6 a$ and $d$ indicate that the lengths of forecast periods do not significantly impact on the GLMPP performance during either the calibration period or the verification period. Figure $6 \mathrm{~b}$ and e suggest that longer analysis window sizes (i.e., $N_{a}=15$ or 20 days) tend to show more consistent results. Figure $6 \mathrm{c}$ and $\mathrm{f}$ seem to show that longer buffer periods (i.e. $N_{f}=30$ or 60 days) provide better agreement between GLMPP results and observations. Figure 7 shows the effects of buffer period lengths on GLMPP reliability. While the CDF curves during the calibration period clearly indicate the superior results of GLMPP adjusted ensemble, longer buffer period length tends to produce more consistent results during the verification period. Longer analysis window and longer buffer periods both play the role of reducing the impacts of wrong initial conditions on the hydrologic simulations during the forecasting periods.

\subsection{Testing GLMPP with different forecast dates}

In this section, we investigate if the GLMPP performance is dependent on the selection of the forecast dates. Again, we used the SAC streamflow data for this purpose. Figure 8 demonstrates how two different forecast dates affect the GLMPP results during both the calibration and verification periods. The two forecasting dates are set at 15 February (Fig. 8a and c) and 15 July (Fig. 8b and d) respectively, with the same settings for data windows: $N_{f}=30$ days, $N_{a}=15$ days, and $N_{\text {buffer }}=60$ days. The results suggest that there is a positive bias for raw streamflow predictions made on 15 February, especially during the early accumulation period, while the over bias is obvious for raw streamflow predictions made on 15 July. The CDF curves based on GLMPP adjusted ensembles seem to be able reduce the biases on both forecast dates. 
(a)

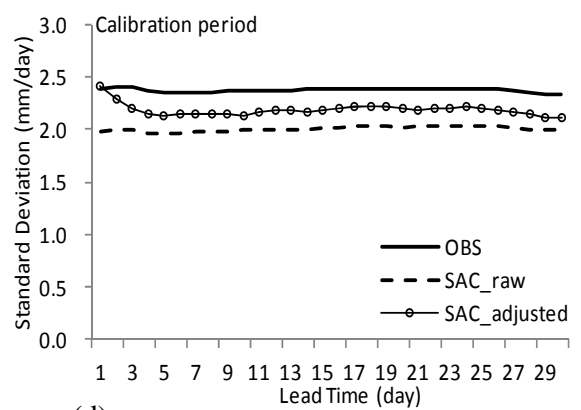

(d)

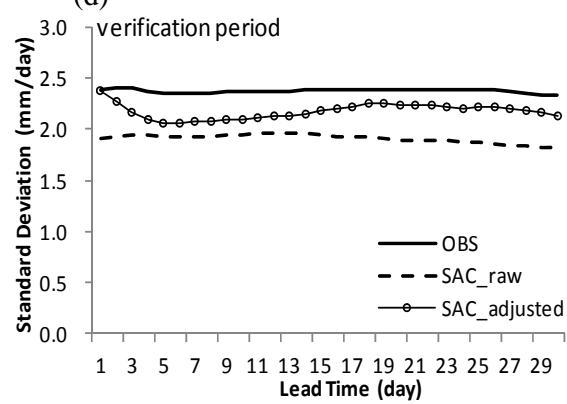

(b)

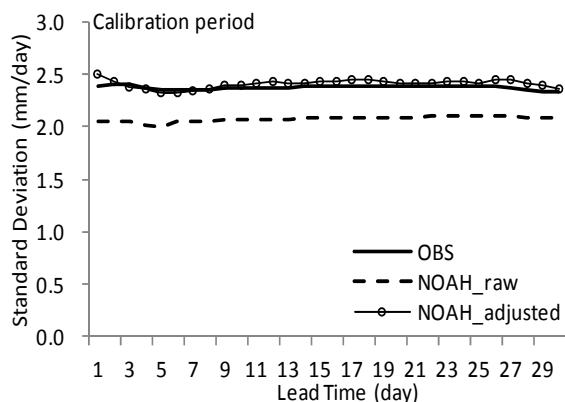

(e)

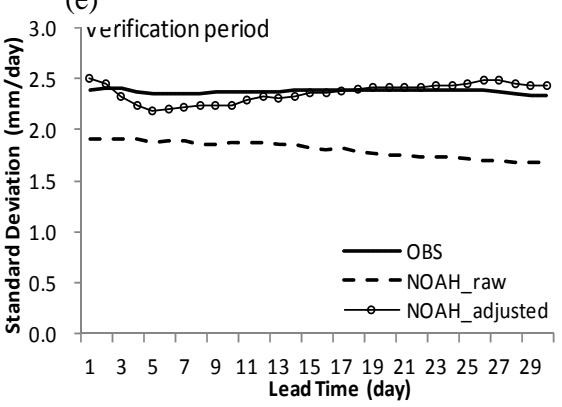

(c)

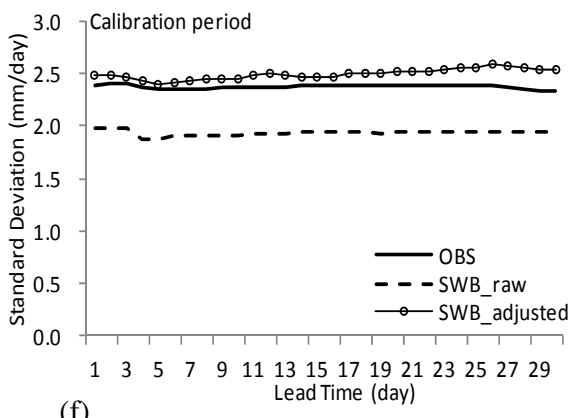

(f)

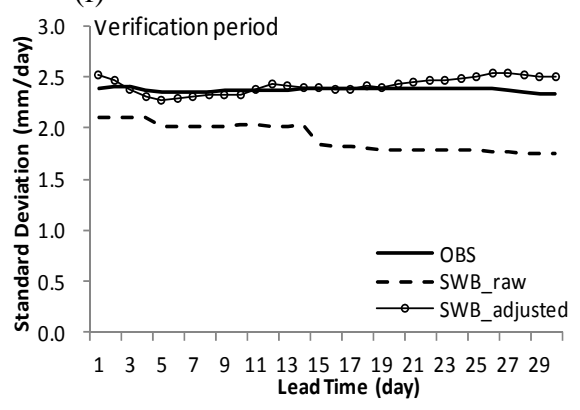

Fig. 4. The mean standard deviations of observed ensemble, raw ensemble, GLMPP adjusted ensemble for the SAC, SWB and NOAH models.

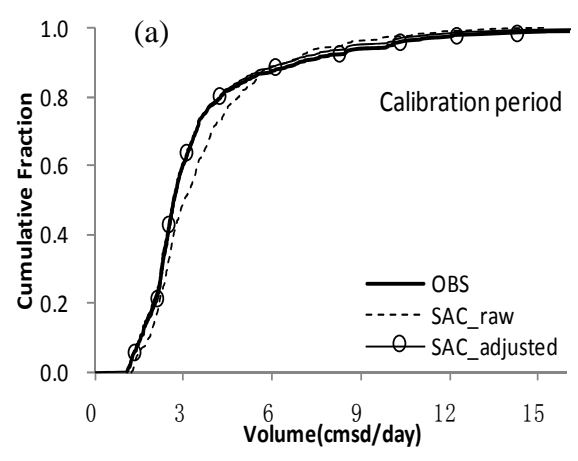

(d)

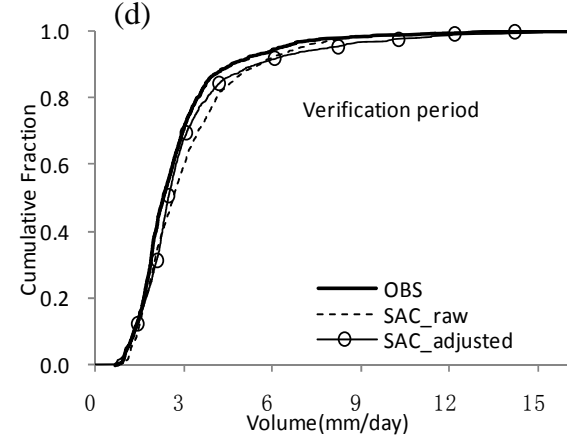

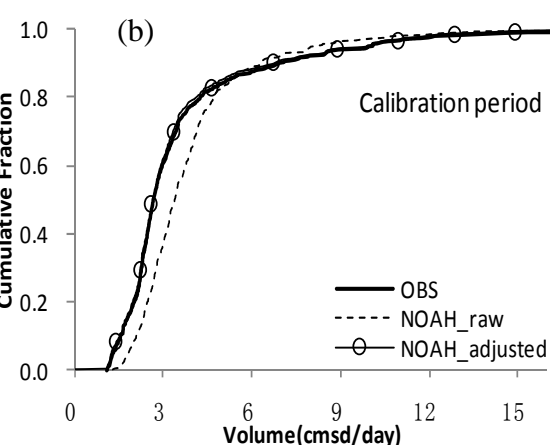

(e)

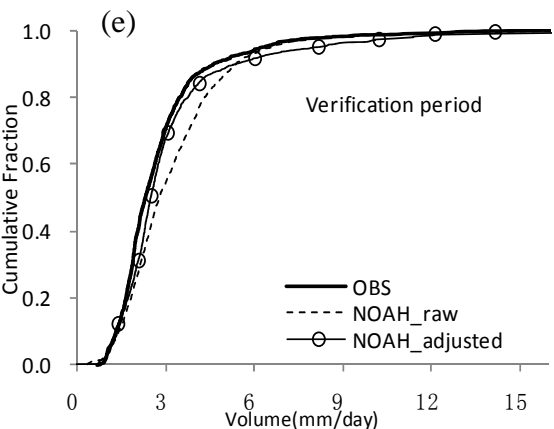

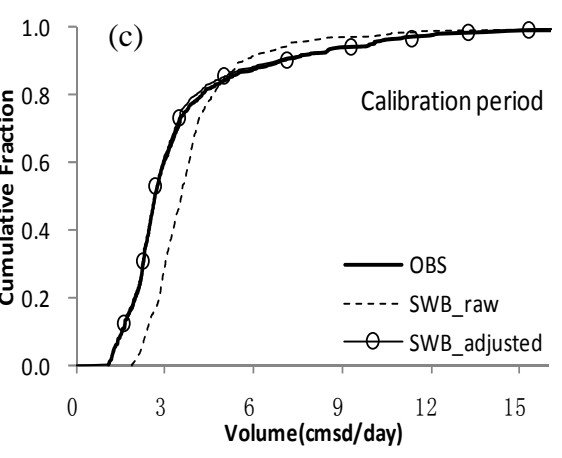

(f)

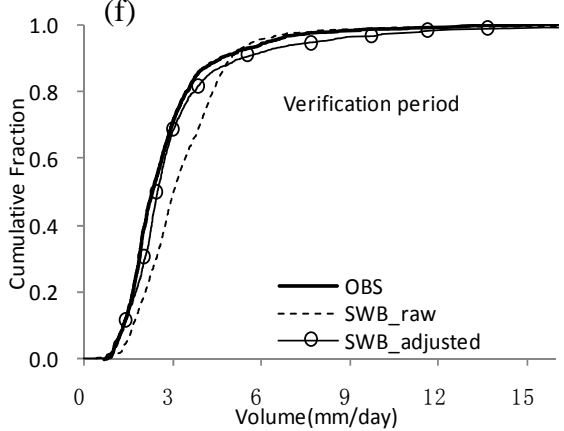

Fig. 5. The CDFs of observed ensemble, raw ensemble and GLMPP adjusted ensemble members for the SAC, SWB and NOAH models. 
(a)

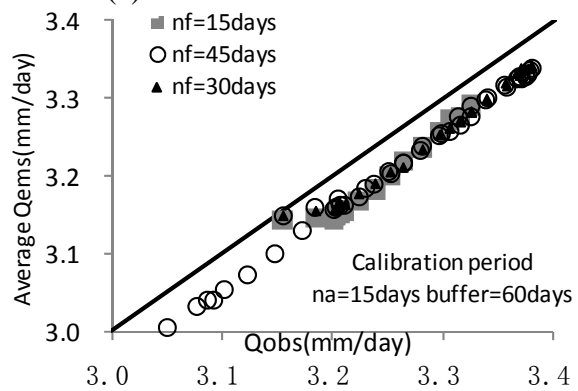

(d)

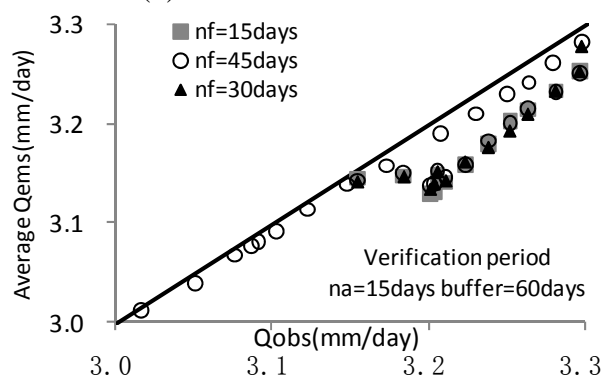

(b)

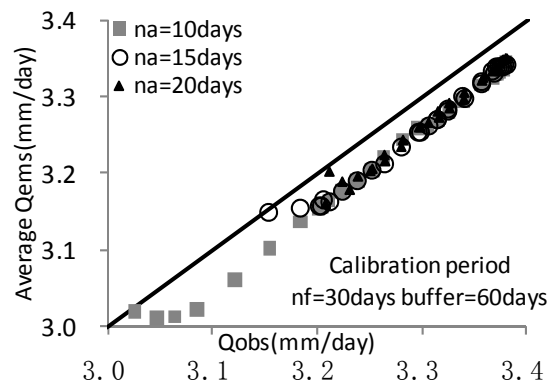

(e)

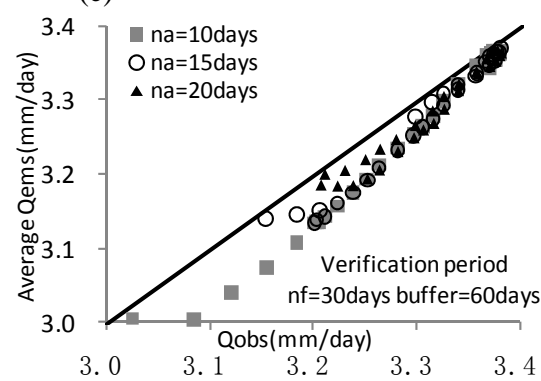

(c)

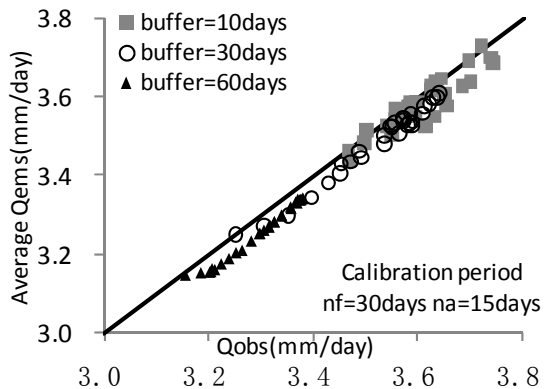

(f)

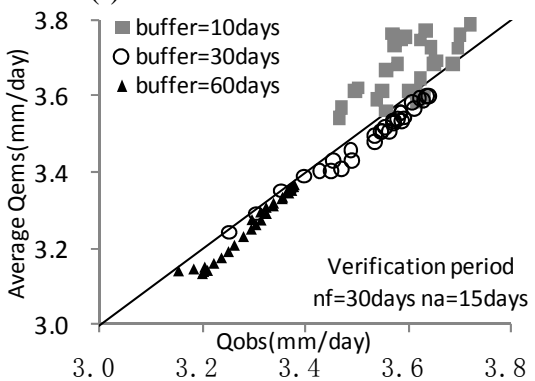

Fig. 6. The effects of different settings for data window on GLMPP results.

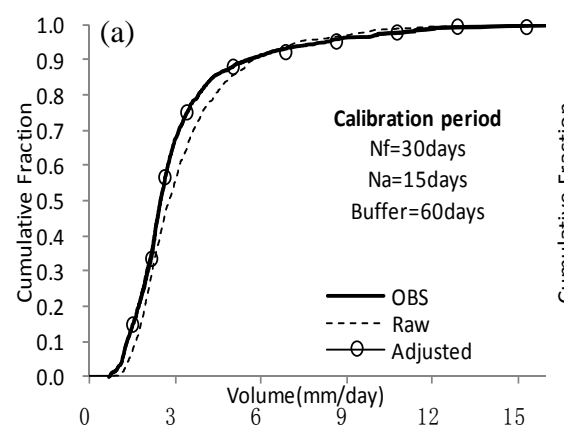

(d)

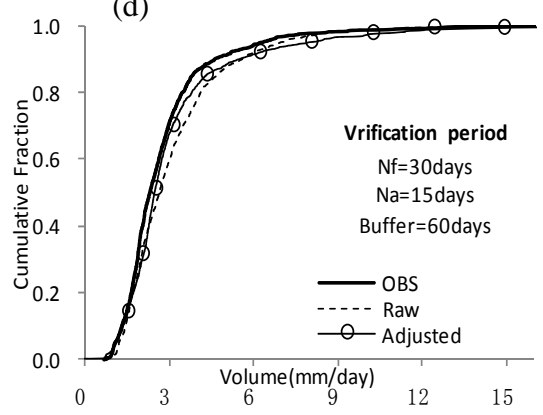

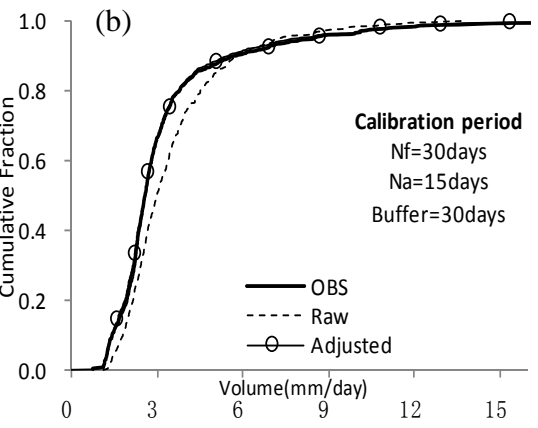

(e)

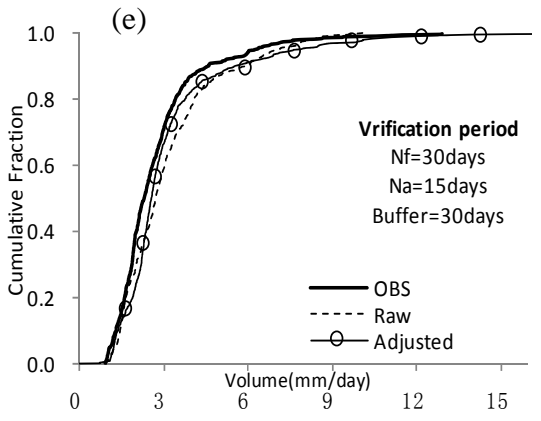

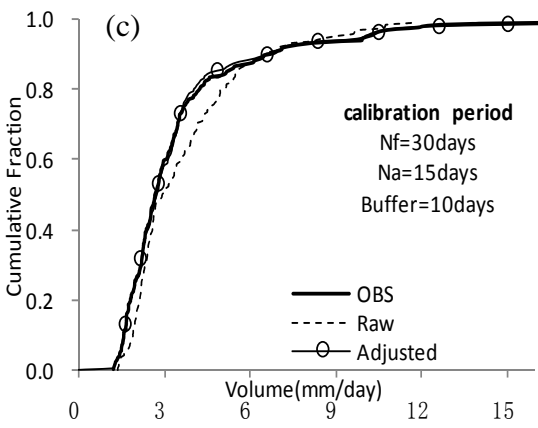

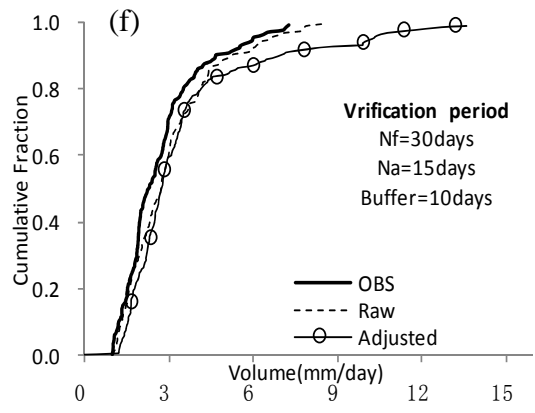

Fig. 7. The effects of buffer period lengths on GLMPP reliability.

\section{Conclusions}

We presented a GLMPP based hydrologic post-processor with the desired properties as presented in Sect. 2. We tested the GLMPP on the SAC, SWB and NOAH models using streamflow data from the Second Workshop on MOPEX. We found that the GLMPP can reduce the mean bias in the streamflow simulations in both the calibration and verification periods. The mean standard deviations of the GLMPP adjusted ensemble are also much closer to the observed ones 

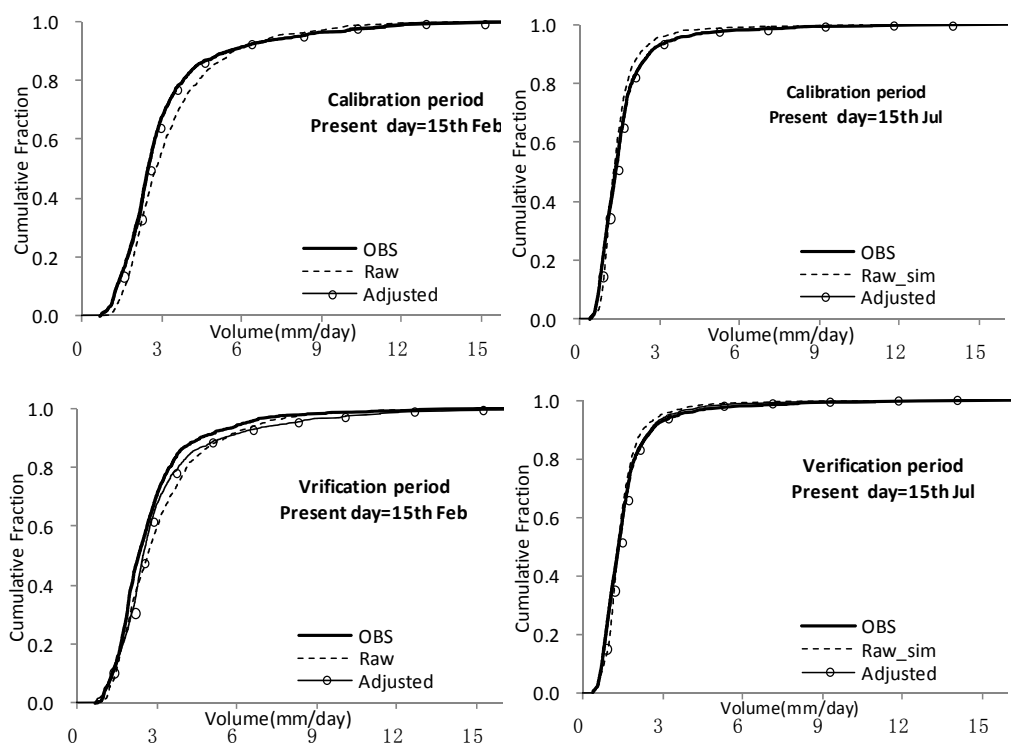

Fig. 8. The CDF curves of the raw, GLMPP adjusted and observed ensembles on two different forecast dates during the calibration and verification periods.

than the raw ensemble. The CDF curves of GLMPP adjusted ensemble agree with the observed CDFs much better than the raw ensemble in both the calibration period and the verification period. Various experiments with the lengths of the analysis period and the length of the buffer period (that control the sample size used for GLMPP calibration) suggest that the GLMPP results can be sensitive to these settings. Particularly, longer analysis window and longer buffer periods tend to provide more consistent results and agree better with observations. The GLMPP seem to work equally well for wetter season (e.g., predictions made on 15 February) and for drier season (e.g., predictions made on 15 July).

This study was done using data from only one basin. The generality of GLMPP can be better proved with inclusion of more basins and with more diverse conditions. The data sample size is limited to 39 years. The results presented in this paper assume that these results would apply to ensemble hydrologic predictions if the meteorological forcing has been calibrated. But testing that assumption is beyond the scope of this limited study.

Acknowledgements. The authors wish to acknowledge the financial support provided by the Chinese Ministry of Science and Technology 973 Basic Research Program Project No. 2010CB428402. We would also like to express our gratitude toward F. Weber and B. Konrad whose reviews greatly enhance the readability of the paper.

Edited by: F. Pappenberger

Reviewed by: two anonymous referees

\section{References}

Andreatis, K. M. and Lettenmaier, D. P.: Assimilating remotely sensed snow observations into a macroscale hydrology model, Adv. Water Resour., 29, 872-886, 2006.

Bogner, K. and Kalas, M.: Error-correction methods and evaluation of an ensemble based hydrologic forecasting system for the Upper Danube catchment, Atmos. Sci. Lett., 9(2), 95-102, 2008.

Beven, K. J. and Binley, A. M.: The future of distributed models: Model calibration and uncertainty prediction, Hydrol. Process., 6, 29-44, 1992.

Burnash, R. J., Ferral, R. L., and McGuire, R. A.: A generalized streamflow simulation system conceptual modeling for digital computers, Tech. Report, US Dept. of Commer. Natl. Weather Serv. and State of Calif., Dept. of Water Resour., 1973.

Clark, M., Gangopadhyay, S., Hay, L., Rajagopalan, B., and Wilby, R.: The Schaake Shuffle: A Method for Reconstructing SpaceTime Variability in Forecasted Precipitation and Temperature Fields, J. Hydrometeor., 5, 243-262, 2004.

Chen, F. and Dudhia, J.: Coupling an advanced landsurface/hydrology model with the Penn State-NCAR MM5 modeling system, Part I: Model implementation and sensitivity, Mon. Weather Rev., 129, 569-585, 2001.

DeMargne, J., Mullusky, M., Werner, K., Adams, T., Lindsey, S., Schwein, N., Marosi, W., and Welles, E.: Application of Forecast Verification Science to Operational River Forecasting in the U.S. National Weather Service, Bulletin of American Meteorological Society, 90(6), 779-784, 2009.

Duan, Q.: Global Optimization for Watershed Model Calibration, in: Calibration of Watershed Models, edited by: Duan, Q., Gupta, H. V., Sorooshian, S., Rousseau, A. N., and Turcotte, R., Water Sci. Appl., 6, 89-104, Washington, DC, 2003.

Duan, Q., Sorooshian, S., and Gupta, V. K.: Effective and Efficient Global Optimization for Conceptual Rainfall-Runoff Models, 
Water Resour. Res., 28(4), 1015-1031, 1992.

Duan, Q., Gupta, H., Sorooshian, S., Rousseau, A., and Turcotte, R. (Ed.): Advances in Calibration of Watershed Models, Water Science and Application Series 6, American Geophysical Union, Washington, DC, 345 pp., 2003.

Duan, Q., Schaake, J., Andreassian, V., Franks, S., Gupta, H. V., Gusev, Y. M., Habets, F., Hall, A., Hays, L., Hogue, T., Huang, M., Leavesley, G., Liang, X., Nasonova, O. N., Noilhan, J.Oudin, L. Sorooshian, S., Wagener, T., and Wood, E. F.: Model Parameter Estimation Experiment (MOPEX): Overview and summary of the second and third workshop results, J. Hydrol., 320(1-2), 3-17, 2006.

Krzysztofowicz, R.: Transformation and normalization of variates with specified distributions, J. Hydrol., 197, 286-292, 1997.

Krzysztofowicz, R.: Integrator of uncertainties for probabilistic river stage forecasting: precipitation-dependent model, J. Hydrol., 249, 69-85, 2001.

Krzysztofowicz, R. and Maranzano, C. J.: Hydrologic uncertainty processor for probabilistic stage transition forecasting, J. Hydrol., 293, 57-73, 2004.

Krzysztofowicz, R. and Sigrest, A. A.: Calibration of probabilistic quantitative precipition forecasts, Weather Forecast., 14, 427442, 1999 .

Kuczera, G. and Parent, E.: Monte Carlo assessment of parameter uncertainty in conceptual catchment models: The Metropolis algorithm, J. Hydrol., 211, 69-85, 1998.

McEnery, J., Ingram, J., Duan, Q., Adams, T., and Anderson, L.: NOAA's Advanced Hydrologic Prediction Service: Building Pathways for Better Science in Water Forecasting, Bull. Amer. Meteorol. Soc., 86(3), 375-385, 2005.

Reggiani, P., Renner, M., Weerts, A. H., and van Gelder, P. A. H. J. M.: Uncertainty assessment via Bayesian revision of ensemble streamflow predictions in the operational river Rhine forecasting system, Water Resour. Res., 45, W02428, doi:10.1029/2007WR006758, 2009.
Schaake, J. C., Koren, V. I., Duan, Q. Y., Mitchell, K., and Chen, F.: Simple water balance model for estimating runoff at different spatial and temporal scales, J. Geophys. Res., 101(D3), 74617475, 1996.

Schaake, J., Franz, K., Bradley, A., and Buizza, R.: The Hydrologic Ensemble Prediction EXperiment (HEPEX), Hydrol. Earth Syst. Sci. Discuss., 3, 3321-3332, doi:10.5194/hessd-3-33212006, 2006.

Schaake, J., Demargne, J., Hartman, R., Mullusky, M., Welles, E., Wu, L., Herr, H., Fan, X., and Seo, D. J.: Precipitation and temperature ensemble forecasts from single-value forecasts, Hydrol. Earth Syst. Sci. Discuss., 4, 655-717, doi:10.5194/hessd-4-6552007, 2007.

Seo, D.-J., Perica, S., Welles, W., and Schaake, J. C.: Simulation of precipitation fields from probabilistic quantitative precipitation forecast, J. Hydrol., 239, 203-229, 2000.

Seo, D.-J., Koren, V., and Cajina, N.: Real-time variational assimilation of hydrologic and hydro meteorological data into operational hydrologic forecasting, J. Hydrometeorol., 4, 627-641, 2003.

Seo, D.-J., Herr, H. D., and Schaake, J. C.: A statistical postprocessor for accounting of hydrologic uncertainty in short-range ensemble streamflow prediction, Hydrol. Earth Syst. Sci. Discuss., 3, 1987-2035, doi:10.5194/hessd-3-1987-2006, 2006.

Slater, A. G. and Clark, M. P.: Snow Data assimilation via an ensemble Kalman filter, J. Hydrometeorol., 7(3), 478-493, 2006.

Valencia, R. D. and Schaake, J. C.: Disaggregation processes in stochastic hydrology, Water Resour. Res., 9(3), 580-585, 1973.

Vrugt, J. A., Gupta, H., Bouten, W., and Sorooshian, S.: A Shuffled Complex Evolution Metropolis algorithm for optimization and uncertainty assessment of hydrologic model parameters, Water Resour. Res., 39(8), 1201, doi:10.1029/2002WR001642, 2003.

Wood, A. W. and Schaake, J. C.: Correcting Errors in Streamflow Forecast Ensemble Mean and Spread, J. Hydrometeorol., 9(1), 132-148, 2008. 